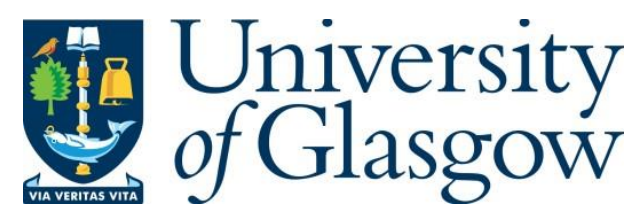

Aidukas, T., Harvey, A. R. and Konda, P. C. (2018) Miniature Fourier Ptychographic Microscope Using Mobile Phone Camera Sensors. Biophotonics Congress: Biomedical Optics Congress 2018, Hollywood, FL, USA, 03-06 Apr 2018. MTu4A.2. ISBN 9781943580415.

There may be differences between this version and the published version. You are advised to consult the publisher's version if you wish to cite from it.

http://eprints.gla.ac.uk/161064/

Deposited on: 23 April 2018

Enlighten - Research publications by members of the University of Glasgow http://eprints.gla.ac.uk 


\title{
Miniature Fourier ptychographic microscope using mobile phone camera sensors
}

\author{
Tomas Aidukas ${ }^{1}$, Andrew R. Harvey ${ }^{{ }^{*}}$, Pavan Chandra Konda ${ }^{1}$ \\ ${ }^{1}$ Imaging Concepts Group, School of Physics and Astronomy, University of Glasgow, Scotland, G12 8QQ, UK \\ *Andy.Harvey@glasgow.ac.uk
}

\begin{abstract}
We report a Fourier ptychographic setup with sub-micron resolution costing around $£ 100$ using mobile phone camera sensors. Reconstruction algorithms were developed to overcome the Bayer pattern on these sensors and robust calibration methods have been developed to tackle alignment errors. (C) 2018 The Author(s)

OCIS codes: (100.5070) Phase retrieval; (170.0180) Microscopy; (110.1758) Computational imaging
\end{abstract}

\section{Introduction}

Fourier ptychographic microscopy (FPM) has gained a lot of interest since it was first proposed in 2013 due to its gigapixel imaging capability using low-NA optics [1,2]. A low-cost setup has been reported using mobile phone camera lenses but its component cost is dictated by the cost of the monochrome sensor for detection and the computer for data acquisition [3]. Here we present a $~ £ 100$ FPM setup equipped with a raspberry pi computer for data acquisition and first implementation of a color sensor for FPM. This is the smallest FPM setup reported, which is also capable of achieving sub-micron resolution within $4 \mathrm{~mm}^{2}$ field-of-view (FOV). The small size and excellent image quality enables imaging inside incubators. Other applications include digital pathology in remote parts of the world where access to high-quality microscopes is limited

A Raspberry Pi computer was chosen for data acquisition due to its low cost and wide opensource community. It has an embedded microcontroller which can be used for controlling LED arrays as well as a camera interface port for a Raspberry Pi camera (a low-cost mobile phone sensor based camera). The lens was separated from the body of the camera and placed further away from the camera in a $3 \mathrm{D}$ printed part to achieve $1.5 \mathrm{X}$ magnification and $0.15 \mathrm{NA}$. This choice was made to achieve twice the Nyquist sampling rate to avoid data loss due to the sparse sampling nature of the color Bayer filter. The camera is mounted on a custom-built translation stage using $0.25 \mathrm{~mm}$ pitch screws for precise translation. A Pimoroni unicorn hat HD is used for illumination, which can be mounted on to the GPIO ports on the Raspberry Pi. The setup is assembled using 3D printed parts as seen in Figure 1 (A). This current setup is controlled by a keyboard and an optical mouse attached to the Raspberry Pi board and a computer monitor is attached for viewing. However, the data acquisition can be controlled remotely using a wireless interface. Moreover, a Raspberry Pi touch screen can also be attached along with a battery pack to make the device completely portable.

A)

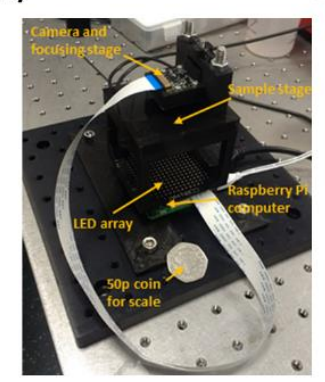

B)

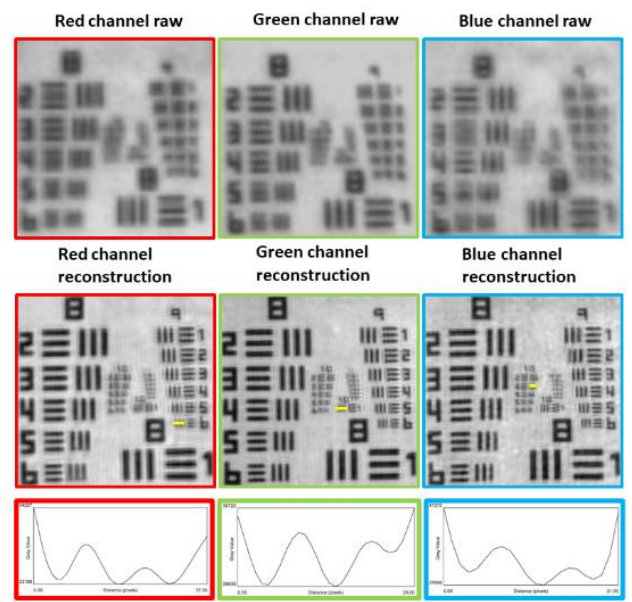

Figure 1 A) Experimental setup B) Images of USAF target indicating resolution improvements after FPM reconstruction compared to the raw images. Line profiles indicate contrast for the maximally resolved bars for all three colors. Red corresponds to the smallest resolution of $1 \mu \mathrm{m}$, while blue can resolve features up to $800 \mathrm{~nm}$

The data from the Raspberry Pi camera is captured in the RAW Bayer format and these images must be pre-processed before use. Conventionally, demosaicing algorithms are used to interpolate image and transform them from grayscale 
to color. However, Fourier ptychography allows using sparsely sampled FPM algorithm to improve reconstruction quality and reduce computational complexity [4]. Interpolated images were used to correct LED position misalignment errors by using a novel self-calibration algorithm [5]. The LED position calibration along with pupil aberration recovery algorithms resulted in high quality reconstruction. In this setup, the data acquisition for multiple colors has been performed sequentially due to light leakage between the RGB channels. This can be done parallelly by using LED multiplexing strategies [6].

\section{Results}

A USAF resolution chart was imaged to validate the resolution improvement and system performance as seen in Figure 1(B). The group 9 features are resolved in all the colors, corresponding to 1-micron features and group 10 features are resolved in green and blue channels, corresponding to 0.8 microns features.
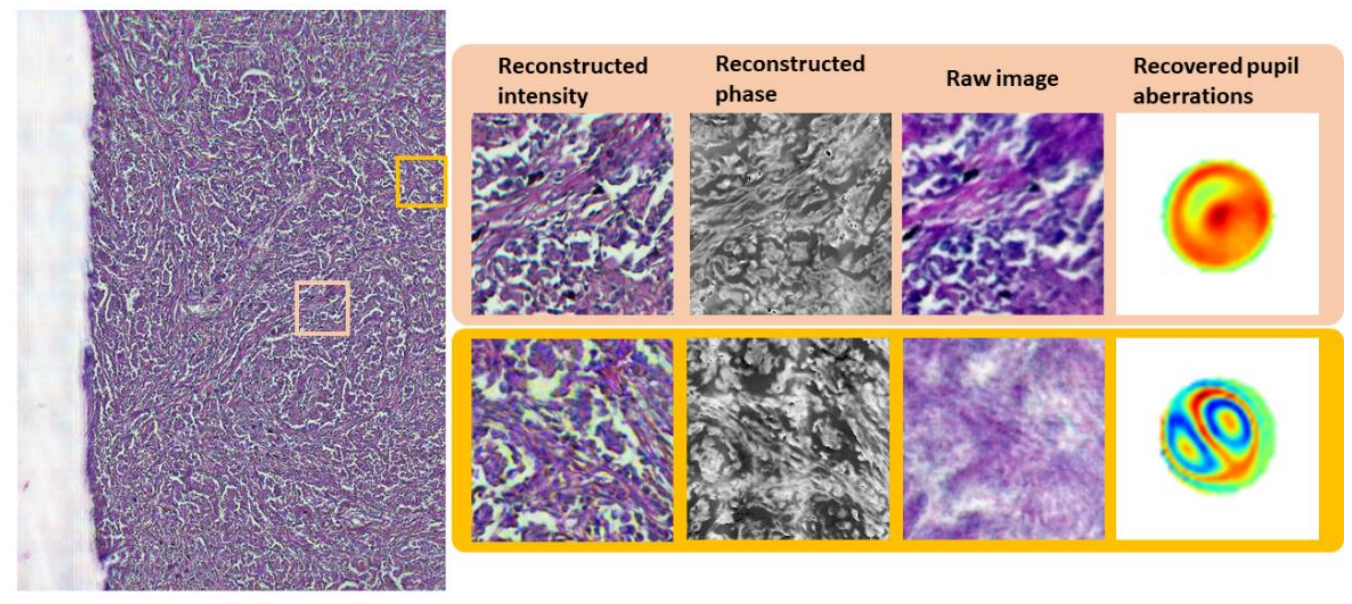

Figure 2 Lung carcinoma pathology sample FPM reconstruction showing intensity phase and pupil aberration recovery. Raw images are shown for comparison to indicate resolution improvement and the severe image degradation due to optical aberrations near the edges.

A lung carcinoma pathology sample was imaged using our system and is shown in Figure 2. It can be observed that the image is reconstructed with high-quality throughout the entire FOV. In addition to the amplitude information, the phase information can also be recovered with our microscope. High amount of aberrations due to the low-cost lens are recovered and corrected within the reconstruction procedure. This device can be extremely useful for pathological applications and can be used in multi-aperture systems [7] for reducing the cost.

\section{References}

1. R. Horstmeyer, G. Zheng, and C. Yang, "Wide-field, high-resolution Fourier ptychographic microscopy.," Nat. Photonics 7, 739-745 (2013).

2. P. C. Konda, J. M. Taylor, and A. R. Harvey, "High-resolution microscopy with low-resolution objectives: correcting phase aberrations in Fourier ptychography," in Proc. SPIE 9630, Optical Systems Design 2015: Computational Optics, 96300X (September 23, 2015); doi:10.1117/12.2191338. (n.d.).

3. S. Dong, K. Guo, P. Nanda, R. Shiradkar, and G. Zheng, "FPscope: a field-portable high-resolution microscope using a cellphone lens," Biomed. Opt. Express 5, 3305 (2014).

4. $\quad$ S. Dong, Z. Bian, R. Shiradkar, and G. Zheng, "Sparsely sampled Fourier ptychography.," Opt. Express 22, 5455-64 (2014).

5. R. Eckert, L. Tian, and L. Waller, "Algorithmic self-calibration of illumination angles in Fourier ptychographic microscopy," in Imaging and Applied Optics 2016, OSA Technical Digest (Online) (Optical Society of America, 2016), Paper CT2D.3. (2016), pp. 3-5.

6. S. Dong, R. Shiradkar, P. Nanda, and G. Zheng, "Spectral multiplexing and coherent-state decomposition in Fourier ptychographic imaging.," Biomed. Opt. Express 5, 1757-67 (2014).

7. P. C. Konda, J. M. Taylor, and A. R. Harvey, "Scheimpflug multi-aperture Fourier ptychography : coherent computational microscope with gigapixels / s data acquisition rates using 3D printed components," in Proc. SPIE 10076, High-Speed Biomedical Imaging and Spectroscopy: Toward Big Data Instrumentation and Management II, 100760R (February 22, 2017) (n.d.). 